\title{
An Introduction to Light Interaction with Human Skin
}

\author{
Gladimir V. G. Baranoski ${ }^{1}$ \\ Aravind Krishnaswamy ${ }^{1}$
}

\begin{abstract}
Despite the notable progress in physically-based rendering, there is still a long way to go before one can automatically generate predictable images of organic materials such as human skin. In this tutorial, the main physical and biological aspects involved in the processes of propagation and absorption of light by skin tissues are examined. These processes affect not only skin appearance, but also its health. For this reason, they have also been the object of study in biomedical research. The models of light interaction with human skin developed by the biomedical community are mainly aimed at the simulation of skin spectral properties which are used to determine the concentration and distribution of various substances. In computer graphics, the focus has been on the simulation of light scattering properties that affect skin appearance. Computer models used to simulate these spectral and scattering properties are described in this tutorial, and their strengths and limitations discussed.
\end{abstract}

Keywords: natural phenomena, biologically and physically-based rendering.

\section{Introduction}

Creating convincing pictures of organic materials, such as human skin, is usually an art entirely left to designers and animators. Recently, in order to overcome these limitations and develop predictive reflectance and scattering models, computer graphics researchers have started to look more closely at the interaction of light with organic materials from a biological point of view. The games and entertainment industries can certainly benefit from being able to automatically generate realistic and predictable images of skin tissues. Currently, the realistic image synthesis of organic materials is several years behind the rendering of inorganic materials. Due to limitations in this area, the non-realistic traits of a synthetic human being are easily perceived despite the sophisticated geometrical models available in the literature.

On the scientific side, the processes of light interaction with human skin have a fundamental importance in biomedical sciences. By studying processes involved in light remission from skin, better protocols can be developed to automatically diagnose medical conditions, such as jaundice (yellowish hue), erythema (redness) [66], as well as tumors at early stages [16]. In the United States, more than 50,000 new cases of melanoma, the most serious form of skin cancer, are reported to the American Cancer Society each year [83]. Understanding

\footnotetext{
${ }^{1}$ Natural Phenomena Simulation Group, School of Computer Science, University of Waterloo, Canada \{gvgbaran@curumin.math.uwaterloo.ca Aravindeinscriber.com\}
} 
how light is absorbed and propagated in skin tissues can assist in the design of lotions protective against harmful solar radiation, and also in the design of superior cosmetics. Hence, the design of biophysically-based and predictive models of light interaction with human skin has applications beyond the scope of computer graphics.

This tutorial discusses the recent advances in the biophysically-based rendering of human skin. In particular, it addresses the processes of light transport and absorption in skin tissues, and examines computer graphics models used to simulate these natural phenomena. Since these models often incorporate algorithms and techniques used in models developed by the biomedical community, an overview of the most influential skin optics modeling approaches used in biomedical applications is also provided. In addition to the direct contributions to the realistic and predictive simulation of skin appearance, the algorithms used in models of light interaction with human skin can be extended to models aimed at the rendering of other organic materials such as hair, fur, leaves, petals, ocular tissues etc...

It is important to note that there are several subsurface scattering models in the graphics literature which are neither biologically motivated nor specifically designed to simulate light interaction with human skin (e.g., plants [6] and hair [55]). An extensive review of these models is beyond the scope of this tutorial, and the interested reader can find more information in a recent survey by Premoze [72]. In addition, there has also been recent works in image based techniques for rendering and altering the appearance of skin [37, 90], which do not address the modeling of light propagation and absorption in skin tissues, and for this reason are not examined in this tutorial.

The remainder of this tutorial is organized as follows. Section 2 presents a concise review of physical parameters used to characterize the appearance of inorganic and organic materials. Section 3 examines the biological factors involved in the light interaction with human skin. Section 4 provides an overview of models of skin optics available in the biomedical field. Sections 5-8 examine the strengths and limitations of models of light interaction with human skin used in image synthesis frameworks. Section 9 outlines a number of issues that need to be addressed to improve the predictability and the applicability of the current models. The tutorial closes with a glimpse of future trends in biophysically-based rendering.

\section{Measurement of Appearance}

The group of measurements necessary to characterize both the color and surface finish of a material is called the measurement of appearance of the material [39]. This group of measurements involves the spectral energy distribution of the propagated light, measured in terms of reflectance and transmittance, and the spatial energy distribution of that light, measured in terms of BRDF (bidirectional reflectance distribution function) and BTDF (bidirectional transmittance distribution function) [65]. 
Greenberg et al. [35] proposed a framework to test, validate and improve the fidelity and efficiency of computer graphics light transport simulations and image display procedures. They emphasized the importance of performing comparisons between simulations and actual measurements so that simulations can be used in a predictive manner. According to their paradigm, it is of fundamental importance that at each stage simulations are compared with measured experiments. Actual measurements of reflectance and transmittance are performed using spectrophotometers, and actual measurements of BRDF and BTDF are performed using goniophotometers $[39,47]$. In order to evaluate models of light interaction with matter or to extract data from previously validated models, one needs to resort to computer simulations of such devices, henceforth called virtual measurement devices.

Spectrophotometry is defined as the quantitative measurement of reflection and transmission properties as a function of wavelength [22], and a spectrophotometer is defined to be any instrument for measuring the spectral distribution of reflected and transmitted radiant power. Spectrophotometers can also be used to determine the absorption characteristics of an object as a function of wavelength. Actual reflectance measurements are usually performed under illuminating and viewing conditions recommended by $\mathrm{CIE}^{2}$. Integrating spheres are used to provide readings where either the illuminant (or viewing) specification is "total" or "diffuse only". The transmittance of translucent specimens depends greatly on the way they are illuminated and mounted in the instrument. Generally transmittance measurements are carried out with the integrating sphere-type spectrophotometers. There are a number of detailed issues specific to performing spectral measurements for translucent materials which are beyond the scope of this course. For a comprehensive discussion of these issues the interested reader is referred to the report by Aydinli and Kaase [4].

Goniophotometry is defined as the measurement of the directional light distribution characteristic of sources, media and materials, and a goniophotometer is defined as an instrument that measures flux (power) as a function of angles of illumination and observation [22]. The measurements made by a goniophotometer can be performed in different ways (Figure 1), and, as a result, there are many possible configurations for these devices. Computer graphics researchers have proposed extensions for industry made goniophotometers [29] as well as designs based on the use of digital cameras $[48,104]$. A reader interested in a detailed description of goniophotometers used in computer graphics is referred to more comprehensive works in this area $[29,57]$.

Baranoski et al. [7] and Krishnaswamy et al. [52] examined in detail the implementation of virtual spectrophotometric and goniophotometric devices respectively. The main question to be addressed when performing a virtual measurement is how many samples should be used. According to the Bernoulli theorem [93], using a sufficiently large number of samples, one will have a high probability of obtaining estimates within the region of asymptotic con-

\footnotetext{
${ }^{2}$ Commission Internationale de L'Eclairage.
} 

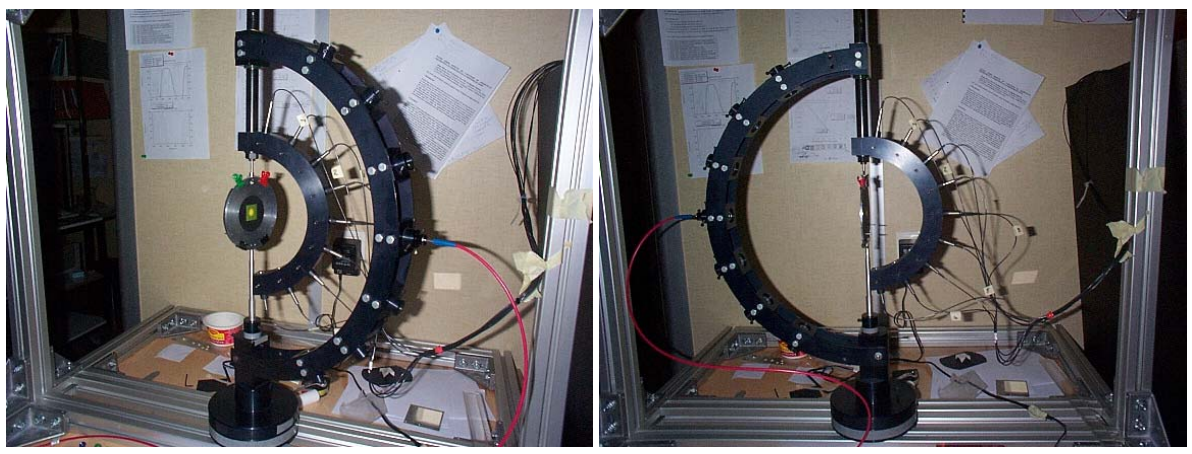

Figure 1. Photographs of a goniophotometer showing different set-ups for BRDF (left) and BTDF (right) measurements. (Courtesy of Stephane Jacquemoud.)

vergence of the expected value of reflectance, or transmittance, being measured. However, as shown by numerical experiments presented by Baranoski et al. [7], the processing time grows linearly with respect to the total number of samples since the cost of the algorithm is constant per sample. In order to minimize the computational costs of virtual spectrophotometric measurements, Baranoski et al. proposed a bound on the number of samples derived from the exponential Chebyshev inequality [76]. For virtual goniophotometric measurements a similar bound can be obtained taking into account the number of patches on the collector sphere used to simulate an actual goniophotometer [52].

\section{Biological Issues}

Skin is a multilayered and inhomogeneous organ (Figure 2). In this section, we outline the biological characteristics of its main constituents, and how they affect the propagation and absorption of light.

\subsection{Structural Characteristics and Spectral Properties}

The first and outermost section of human skin is the stratum corneum, which is a stratified structure approximately 0.01-0.02 $\mathrm{mm}$ thick $[2,59]$. There are skin structural models, however, that consider it part of another tissue, namely the epidermis [91] (Figure 2). The stratum corneum is composed mainly of dead cells, called corneocytes, embedded in a particular lipid matrix [84]. Light absorption is low in this tissue, with the amount of transmitted light being relatively uniform in the visible region of the light spectrum [24].

The epidermis is a $0.027-0.15 \mathrm{~mm}$ thick structure $[2,20,59]$ composed of four layers 


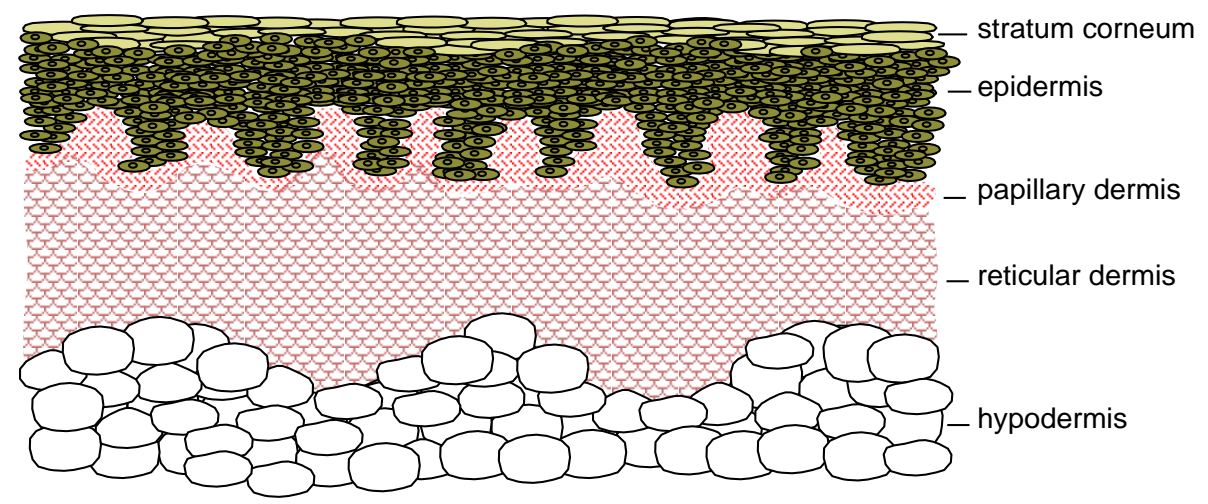

Figure 2. Schematic cross-section of skin tissues and the subcutaneous tissue (hypodermis).

(stratum basale, stratum spinosum, stratum granulosum and stratum lucidum). The epidermis propagates and absorbs light. The absorption property comes mostly from a natural pigment (or chromophore), melanin. There are two types of melanin, the red/yellow phaeomelanin and a brown/back eumelanin [86]. Their absorption spectra are broad (Figure 3), with higher values for shorter wavelengths. The skin color is mostly associated with eumelanin [86]. The ratio between the concentration of phaeomelanin and eumelanin present in human skin varies from individual to individual, with much overlap between skin types [86]. Recent studies reported values between 0.049 and 0.36 [67]. Melanin is produced by cells called melanocytes occurring in the stratum basale, and it is found in membranous particles called melanosomes. The melanin absorption level depends on how many melanosomes per unit volume are in the epidermis. Typically, the volume fraction of the epidermis occupied by melanosomes varies from $1.3 \%$ (lightly pigmented specimens) to $43 \%$ (darkly pigmented specimens) [42].

The dermis is a 0.6-3 $\mathrm{mm}$ thick structure [2, 20,59] which also propagates and absorbs light. It can be divided into two layers: the papillary dermis and the reticular dermis (Figure 2). These layers are primarily composed of dense, irregular connective tissue with nerves and blood vessels (smaller ones in the papillary, and larger ones in the reticular dermis). The volume fraction of blood in tissue can vary, roughly in the $0.2-7 \%$ range $[27,42]$. The fluence rate of blood decreases as we get deeper into the skin, following an almost linear pattern in the dermis [97]. In the blood cells we find another natural chromophore, hemoglobin, which absorbs light and gives blood its reddish color. Normally, the hemoglobin concentration in whole blood is between 134 and $173 g / L$ [106]. In the arteries, 90-95\% of hemoglobin is oxygenated, and in the veins, more than $47 \%$ of the hemoglobin is oxygenated [3]. These two types of hemoglobin, namely oxygenated and deoxygenated hemoglobin, have slightly 
different absorption spectra (Figure 3). Two other blood borne pigments are found in the dermis, bilirubin and $\beta$-carotene, which contribute to the yellowish or olive tint of human skin (Figure 3 ). We remark that $\beta$-carotene may be also found in the epidermis and stratum corneum $[1,54]$.

The hypodermis is an adipose tissue characterized by a negligible absorption of light in the visible region of the spectrum [27]. It is usually not considered part of the skin, and its size varies considerably throughout the body. It can be up to $3 \mathrm{~cm}$ thick in the abdomen and absent in the eye lids. The hypodermis presents significant deposits of white fat, whose cells are grouped together forming clusters. Due to the presence of these white fat deposits, most of the visible light that reaches this tissue is reflected back to the upper layers [20].

\subsection{Scattering Profile}

The scattering profile of human skin has two main components: surface and subsurface scattering. Surface scattering follows Fresnel equations [82], and it is affected by the presence of folds in the stratum corneum. The aspect ratio of these mesostructures depends on biological factors such as aging and hydration [84, 85]. Approximately 5-7\% of the light incident (over the entire spectrum) on the stratum corneum is reflected back to the environment [91]. The remaining portion is transmitted to the internal tissues. Besides the reflectiverefractive scattering caused by the reflection and refraction of light at cellular boundaries, two other types of scattering occur within the skin layers: Mie and Rayleigh scattering [42].

The stratum corneum and the epidermis are characterized as forward scattering media [9]. In the former this behavior is due to the alignment of the fibers, while in the later it is due to Mie scattering caused by particles that are approximately the same size of the wavelength of light (e.g., cell organelles). The level of forward scattering for these tissues is wavelength dependent. Bruls and van der Leun [9] performed goniometric experiments for five wavelengths for both the stratum corneum and the epidermis, and they showed that the scattering profiles are broader towards the shorter wavelengths.

In the dermis, collagen fibers (approximately $2.8 \mu \mathrm{m}$ in diameter and cylindrical [42]) are responsible for Mie scattering, while smaller scale collagen fibers and other micro-structures are responsible for Rayleigh scattering [42]. Light gets scattered multiple times inside the dermis before it is either propagated to another layer or absorbed. This means that the spatial distribution of the light scattered within the dermis quickly becomes diffuse [2]. In fact, Jacques et al., [43] showed through goniophotometric measurements that backscattered light from the dermis is diffuse. While Mie scattering produces variations on both ends of the visible region of the light spectrum, Rayleigh scattering, being inversely proportional to the wavelength of light $\left(\approx \lambda^{-4}\right)$, produces larger variations on the low end of the light spectrum $[42,33]$. 

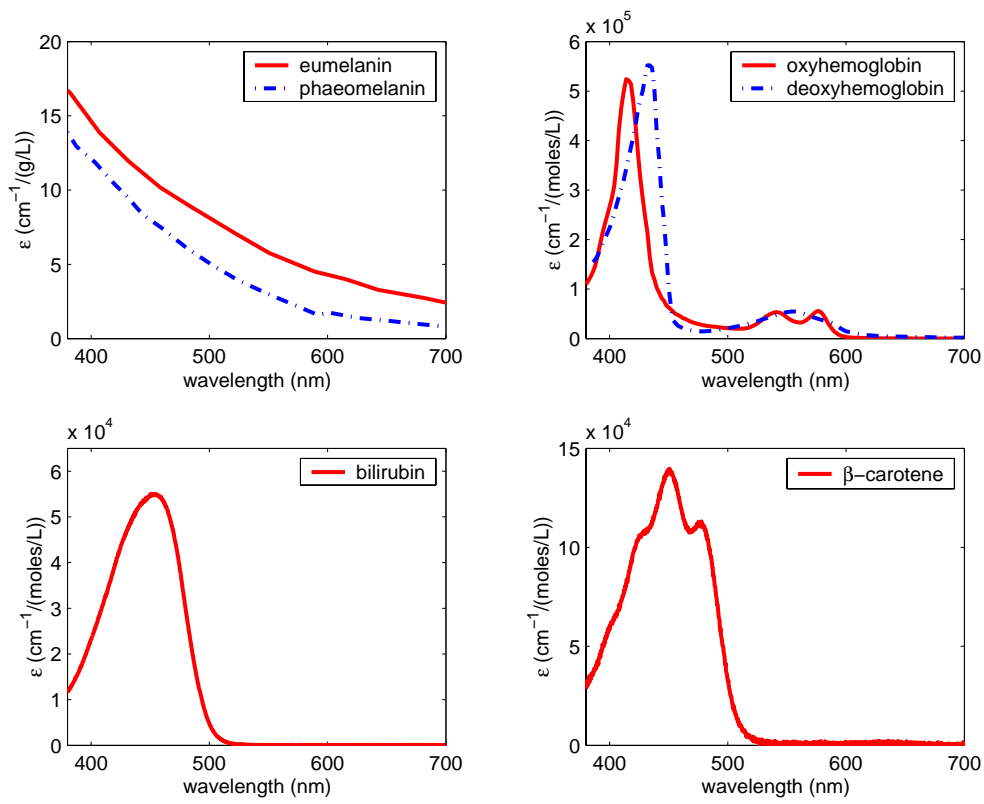

Figure 3. Spectral extinction coefficient curves for pigments present in skin tissues. Courtesy of S. Prahl and the Oregon Medical Laser Center. Absorption spectra are obtained by multiplying each curve by the pigment's molecular weight and concentration.

\section{Review of Models Used in Biomedical Applications}

In this section we provide an overview of relevant modeling approaches and models used in biomedical applications involving tissue optics, and group them according to their similarities. For a comprehensive literature review on this topic, the reader is referred to the texts by Cheong et al. [13] and Tuchin [91]. It is worth noting that these models are mostly aimed at the measurement and reproduction of skin spectral properties to determine the content and distribution of various substances $[89,111]$, i.e., scattering properties affecting skin appearance are usually not addressed. Moreover, a substantial portion of the work done by the biomedical community is either laser-based or aimed at wavelengths outside the visible region of the light spectrum. 


\subsection{Kubelka-Munk Theory Based Models}

In the beginning of the century, Kubelka and Munk [53] developed a simple relationship between the scattering and absorption coefficients ${ }^{3}$ of paint and its overall reflectance. This relationship is known as the Kubelka-Munk theory (henceforth referred to as K-M theory). It applies energy transport equations to describe the radiation transfer in diffuse scattering media using two parameters: the scattering and the absorption coefficients. The K-M theory, as originally stated, is considered to be a two-flux theory, since only two types of diffuse radiant flux are involved, namely a diffuse downward flux and a diffuse upward flux. The relations between the fluxes are expressed by two simultaneous linear differential equations [53]. The original K-M theory also assumes that the medium (specimen) presents inhomogeneities which are small compared to its thickness.

The K-M theory based models (henceforth referred to as K-M models), used in biological tissue optics, also called flux models [13], use K-M equations relating tissue optical properties to measured reflectance and transmittance. Although they are based on the two-flux K-M theory, these models expanded the original K-M formulation by adding more coefficients and/or fluxes. For example, van Gemert and Star [98] included a phase function $^{4}$, optical depth and the effective albedo in their K-M model. They used a phase function consisting of a combination of a forward peaked and a symmetric scattering to represent the tissue's expected experimental scattering behavior. In the original K-M theory formulation [53], the albedo was used to represent the fraction of diffuse light reflected by a matte, plane parallel coating of a given thickness. The effective albedo used by van Gemert and Star [98], however, represents the ratio between the scattering coefficient and the total attenuation coefficient, which is given by the sum of the absorption coefficient and the scattering coefficient [40]. In the context of this tutorial, unless otherwise stated, albedo refers to the definition used by van Gemert and Star [98].

Tuchin et al. $[92,107]$ used a four-flux model composed of the two diffuse fluxes used in the original K-M theory, and two collimated laser beams, the incident one and the one reflected from the bottom boundary of the specimen. Yoon et al. $[110,108]$ used a seven flux model to obtain a three dimensional representation of the scattered radiation caused by an incident laser beam in a semi-infinite medium (infinite in $\mathrm{x}$ and $\mathrm{y}$, but finite in $\mathrm{z}$ ).

In skin optics, the K-M theory was initially applied to specific skin tissues. Anderson and Parish [2] used a K-M model to compute absorption and scattering coefficients for the dermis tissues. Wan et al. [102] extended this model to compute the absorption and scat-

\footnotetext{
${ }^{3}$ The absorption and scattering coefficients represent the product of the actual absorption (or scattering) cross section by the density of the absorbers (or scatterers) [40]. The absorption and scattering cross sections of a particle have the dimension of area, and, generally, they are functions of the orientation of the particle and the state of polarization of the incident light [95].

${ }^{4} \mathrm{~A}$ phase function represents the directional scattering of the light incident onto a particle [95].
} 
tering coefficients for the epidermis tissues, taking into account both collimated and diffuse incident irradiance. In both cases $[2,102]$, the forward scattering in the epidermis was not considered. Diffey [19] proposed a K-M model which added two features to the previous models, namely it takes into account forward and backward scattering and allows changes in the refractive index at the air/skin interfaces. Cotton and Claridge [17] proposed a model to determine the color of human skin which applies the K-M equations to the dermis layer. This model takes into account the presence of melanin and blood pigments. Recently, Doi and Tominaga presented a model which considers the skin composed of two layers: epidermis and dermis. They apply the K-M theory to both layers. Their model provides weights for five skin pigments (melanin, carotene, oxy-hemoglobin, deoxy-hemoglobin and bilirubin) as well as the skin surface reflectance. These six parameters are obtained by fitting the estimated reflectance to measured values using the least squares method [10].

Although the K-M theory allows a simple quantitative treatment of skin spectral properties and recent extensions to the original two-flux theory have improved its applicability to biological tissue optics, it is not a thorough model of optical radiation transfer. The K-M models can be considered analytical, and they allow the rapid determination of skin optical parameters through inversion procedures ${ }^{5}$. However, the relative simplicity and speed of these models are achieved at the expense of accuracy [91], which requires a more detailed analysis of the structure and optical properties of the different skin tissues.

\subsection{Diffusion Theory Based Models}

Photon propagation in optically turbid media, such as skin tissues, can be described using the Boltzmann photon transport equation [40], which requires the optical properties of the medium be expressed in terms of scattering coefficient, absorption coefficient and phase function [21]. Diffusion theory can be seen as an approximate solution of this equation, i.e., it combines the scattering and the phase function in one parameter, called reduced scattering coefficient.

Models based on the diffusion approximation [99] or combined with other approaches, such as the K-M theory [98, 97] or Monte Carlo methods [103], have been used in biomedical investigations involving light propagation in turbid media. Farrell and Patterson [25] proposed a model based on the diffusion theory to be used in the non-invasive determination of the absorption and scattering properties of mammalian tissues. Their model incorporates a photon dipole source in order to satisfy the tissue boundary conditions. Recently, Doornbos et al. [21] proposed a method based on the diffusion theory for measuring optical properties and deriving chromophore concentrations from diffuse reflection measurements at the surface

\footnotetext{
${ }^{5}$ An inversion procedure is a way to derive biochemical and optical properties from in situ and non-destructive experiments [31]. "Inversion" implies a reversal of the usual process of calculating reflection and transmission, i.e., reflectance and/or transmittance values are used as input instead of output.
} 
of a turbid media.

Models based on the diffusion theory are amenable to analytic manipulation, place minor constraints on the type of sample and are relative easy to use [71]. The diffusion theory, however, can be applied only when scattering events are more probable than absorption events. This is usually the case for mammalian tissues in the red and near infrared regions of the light spectrum[28]. Not surprisingly, diffusion models have been used in medical applications involving red lasers $[99,109]$. When the absorption coefficient of a turbid medium is not significantly smaller than the scattering coefficient, the diffusion theory provides a poor approximation for the photon transport equation [71, 74, 109].

\subsection{Radiative Transport Models}

The K-M and diffusion theories mentioned in the previous sections can be seen as special cases of radiative transfer phenomena. When non-stochastic accurate solutions of the radiative transport equation in biological tissues are required, more robust methods need to be used, e.g., the successive scattering technique, Ambartsumian's method, the discrete ordinate method, Chandrasekhar's X and Y functions and the adding-doubling method [69]. Their applicability, however, is usually limited to simple conditions and slab geometries ${ }^{6}$. A comprehensive review of these methods is beyond the scope of this work, and the interested reader is referred to the texts by van de Hulst [94] and Prahl [69]. It is worth noting, however, that the adding-doubling method has several advantages with respect to the other techniques. It permits asymmetric scattering, arbitrarily thick samples, Fresnell boundary conditions, and relatively fast computation [69].

The adding method requires that the reflection and transmission of two slabs be known. They are used to compute the reflection and transmission of another slab comprised of these two individual slabs. In its original definition, the doubling method corresponds to the special case in which both slabs are identical [94]. Later on, it was extended to include the addition of two non-identical slabs [69]. Once the transmission and reflection for a thin slab are known, the reflection and transmission for a target slab can be computed by doubling the thickness of the thin slab until it matches the thickness of the target slab.

Prahl et al. [71] applied an inverse adding-doubling method (IAD: "inverse" implying its use as an inversion procedure) to determine the scattering, absorption coefficient and the asymmetry factor ${ }^{7}$ of biological tissues. The IAD is an iterative method which consists of guessing a set of optical properties, calculating the reflection and transmission using adding-doubling method, comparing the calculated values with the measured reflection and

\footnotetext{
${ }^{6}$ In the tissue optics context, a "slab" refers to an infinite plane parallel layer of finite thickness [69].

${ }^{7}$ The asymmetry factor correspond to the mean cosine of the scattering angles [95]. It also corresponds to the asymmetry parameter of phase functions.
} 
transmission, and repeating the process until a match is obtained. This method may be used when the propagation of light through the specimen can be described by the one-dimensional radiative transport equation. The accuracy of this method, however, depends on the criteria applied to define a "sufficiently thin slab" [69]. There are also restrictions on the sample geometry, i.e. it must be an uniformly illuminated and homogeneous slab [71].

\subsection{Monte Carlo Based Models}

The Monte Carlo method was originally proposed by Metropolis and Ulam [61] to simulate radiative transfer processes through a stochastic model which consists of keeping track of photon histories as they are scattered and absorbed in a given medium. The core of Monte Carlo models of light transport in turbid media is represented by the scattering profile of the particles, which can be described by a phase function [69].

In 1988, Prahl [69] proposed a Monte Carlo based algorithm to model light transport in tissue during laser irradiation. Although a Monte Carlo based approach was used before to study light propagation in tissue [105], Prahl's Monte Carlo algorithm, to the best of our knowledge, was the first to incorporate a phase function, namely the Henyey-Greenstein phase function or HGPF [38], to represent the scattering profile of skin tissues. Recently, however, the applicability of the HGPF to the simulation of light interaction with biological tissues have started to be questioned [5, 62]. It is worth noting that the HGPF is neither based on a mechanistic theory of scattering [43], nor does it have a biological basis.

Monte Carlo models have been extensively used to simulate biological tissue optics [91] since they can provide a flexible, and yet rigorous approach to this problem [103]. These models can be easily implemented, and they are sufficiently flexible to allow the simulation of complex tissues. Theoretically, Monte carlo solutions can be obtained for any desired accuracy [69]. In practice, the accuracy of Monte Carlo simulations is bounded by the accuracy of the input parameters and the use of proper representations for the mechanisms of scattering and absorption of photons. To the best of our knowledge, the Monte Carlo models used in biomedicine [14, 59, 60, 70, 75, 77], colorimetry [89] and pattern recognition [63] provide only reflectance and transmittance readings for skin samples, i.e., BRDF and BTDF quantities for the whole skin are not computed. We remark that these models are mostly aimed at laser applications, and comparisons of modeled reflectance and transmittance values with actual measured values are scarce. 


\section{The H-K Multiple-Layer Scattering Model}

\subsection{Overview}

Hanrahan and Krueger [36] proposed a model to simulate subsurface reflection and transmission from layered surfaces, known as the H-K multiple-layer scattering model. This intuitive idea of a layered surface model has appeared several times in fields such as remote sensing [41] and biomedicine (Section 4). This model can be used to simulate the scattering profile of layered materials appearing in nature, such as biological tissues (e.g., skin, leaves etc.) or inorganic materials (e.g., snow, sand etc.). In this tutorial, it is examined in the context of the rendering of human skin, which was modeled by Hanrahan and Krueger as two layers, epidermis and dermis. Recently, $\mathrm{Ng}$ and Li [64] proposed an extension to the $\mathrm{H}-\mathrm{K}$ model which consists in adding a sebum layer on the top of the epidermis layer.

The scattering simulation algorithm used in the H-K model is based on the onedimensional transport theory which is applied in conjunction with a Monte Carlo sampling scheme. Transport theory is a heuristic approach based on abstracting microscopic parameters into statistical averages. It also forms the computational framework for solving the rendering equation [36]. Hanrahan and Krueger assume planar surfaces, and use Fresnel coefficients to find how much light will pass through the outermost surface of the coating. The model then evaluates the scattering and absorption within each layer, including the reflection and transmission effects at each internal boundary. The BRDF and BTDF are then described by a combination of the reflection function on the outer surface and the internal subsurface scattering handled by the Monte Carlo evaluation. We remark that the Monte Carlo algorithm used by the H-K model to implement directional scattering within a layer was originally proposed by Prahl [69] to investigate laser irradiation in tissue (Section 4.4), and its formulation is described in detail by Prahl et al. [70].

In the H-K model, it is assumed that if a material is a mixture of several materials, then the mixture can be considered to be an uniform and homogeneous combination whose properties are given by a sum of the descriptors of the components weighted by percentages. The material descriptors include the index of refraction, the absorption cross section, the scattering cross section, the depth (or thickness) and a phase function (the HGPF). The absorption and scattering cross sections used by Hanrahan and Krueger correspond in fact to the volumetric absorption and scattering coefficients respectively [69]. In this tutorial, for the sake of consistency with the tissue optics literature, we use the terms absorption coefficient and scattering coefficient instead of the terms absorption cross section and scattering cross section used by Hanrahan and Krueger [36]. In the context of H-K model, these coefficients may be interpreted as the probability per unit length of an interaction of a particular type [36]. 


\subsection{Strengths and Limitations}

The H-K multiple-layer model has the merit of being one of the first computer graphics models to address important issues related to the simulation of light interaction with biological materials. However, because of its generality, it tends to overlook important specific characteristics and properties of organic materials, such as the mechanisms of absorption of light by natural pigments and their specific absorption coefficients. In the H-K model, these aspects are considered only implicitly through the use of coefficients available in the biomedical literature, which, in turn, were obtained using inversion procedures (Section 4.1). Hence, the reflectance and transmittance of skin specimens are not computed directly, but implicitly introduced into the model as the albedo (the ratio between the scattering coefficient and the attenuation coefficient). In other words, the H-K multiple-layer model has to be considered as a scattering model, instead of a reflectance model, since reflectance and transmittance values are not provided by the model.

Hanrahan and Krueger suggest that their model could be combined with the TorranceSparrow model [87] in order to take into account the surface reflection on the outermost layers. The latter was, however, designed based on experimental data for inorganic materials, and its parameters are not biologically meaningful. Thus, it is not clear what criteria should be used in the selection of its parameters in order to model light interaction with organic materials. The use of the HGPF in the subsurface scattering simulation of skin tissues raises some issues as well. First, its main parameter, the asymmetry factor, has no direct connection with the underlying biophysical phenomena. Second, the HGPF was initially meant to be used in tissue optics just as a function to fit multiple scattering data of skin measured at specific wavelengths by Bruls and van der Leun [9] (Section 3.2). Recently, Baranoski et $a l$. [5] demonstrated that the HGPF approximations deviate from the measured data, and its generalized application to any organic tissue at any wavelength may lead to incorrect results, specially using asymmetry factors determined by fitting the HGPF to specific data sets that may have no relationship with the material at hand.

The evaluation of the H-K multiple-layer model was based solely on visual inspection of computer generated images, which precludes an analysis of the accuracy of the BRDF values provided by this model. Hanrahan and Krueger generated images of a human face, whose different colors were rendered using texture maps to arbitrarily control pigment coefficients, i.e., actual absorption spectra of different skin pigments were not applied. Hanrahan and Krueger compared images rendered using the H-K model with images rendered using a Lambertian model. They also generated a test image in which they added a thin layer of oil on the skin to provide an additional term to their model. Although the H-K model can be used to render believable images of human skin, its accuracy was not sufficiently evaluated to classify it as a predictive model [36]. 


\section{The Discrete-Ordinate Model}

\subsection{Overview}

The discrete-ordinate model proposed by Stam [78], henceforth referred to as D-O model, assumes that the skin is composed of a layer with constant optical properties bounded by two isotropic rough surfaces. It is assumed that the skin has an uniform index of refraction, and it is bounded above and below by media having uniform indices of refraction as well. Following Hanrahan and Krueger [36], the D-O model assumes that the skin depth is along the $z$-direction and the skin properties are uniform in each $x y$-plane. The parameters used to model the skin layer are the optical depth, the albedo and the asymmetry factor of the phase function (the HGPF). It also uses a parameter representing the roughness of the surfaces bounding the skin layer, which affects the reflection and transmission at these layers. Each parameter used in the D-O model is dimensionless, and varies from zero to one.

In order to model a skin layer bounded by rough surfaces, Stam extended the work by Stamnes and Conklin [79] for a skin layer bounded by a smooth surface, which is based on the discrete-ordinate approximation of radiative transfer equation [11]. The method of discreteordinates divides the radiative transport equation into $n$ discrete fluxes to obtain $n$ equations with $n$ unknowns [69]. These equations are solved numerically by Stam using Fourier transforms and eigenanalysis. His approach was inspired by the work of Jin and Stammes [46]. Furthermore, Stam takes into account the reflection and refraction from the rough surfaces by extending the BRDF model proposed by Cook and Torrance [15] and following the work of van Ginneken et al. [100] in which the surfaces are assumed to have a normal distribution of heights.

The discrete representations of the BRDF and BTDF of the skin layer form a collection of matrices that are precomputed for different values of the parameters used in the D-O model. This precomputation generates a large data set. In order to allow a practical use of this data in rendering frameworks, Stam compressed it using an approximation based on cosine lobes. The cosine terms were chosen by visually comparing the data to the approximation. The data set was then further compressed by fitting a cubic Bèzier surface to the data stored in the reflection and transmission matrices. The control vertices of the Bèzier surfaces were constrained to respect their symmetry, i.e., to obey the Helmholtz reciprocity rule ${ }^{8}$.

\subsection{Strengths and Limitations}

The D-O model is only a scattering model since reflectance and transmittance quantities are not computed. Although it is biologically-motivated, it does not take into account

\footnotetext{
${ }^{8}$ This condition states that the BRDF for a particular point and incoming and outgoing directions remains the same if these directions are exchanged [65].
} 
the structural characteristics of skin tissues and the biological processes that affect propagation and absorption of light in these tissues. The oversimplification of these biological processes, however, is not accompanied by the mathematical complexity of the algorithms used in the D-O model. Although these are not as complex as the rare analytical solutions for radiative transfer problems found in the literature, they are certainly less straightforward than the algorithms used in Monte Carlo based models. The main advantage of the D-O model over stochastic approaches using Monte Carlo methods is speed, which is sustained using precomputation and compressing schemes. We remark that the outputs of Monte Carlo based models can also be precomputed and compressed off-line, and reconstructed during rendering, reducing their operational costs.

On a rough surface it is possible that some points will not receive light, a phenomenon known as shadowing effect. The D-O model takes into account this phenomenon, which is rarely incorporated in computer graphics models of light interaction with organic materials. As mentioned above, in order to accomplish that, Stam extended the Cook and Torrance [15] using a shadowing function proposed by van Ginneken et al. [100]. It is worth noting that both models were mainly aimed at inorganic or man made materials, and their applicability to biological materials has not been verified.

The discrete-ordinates approach to solve radiative transfer problems is suitable when the material phase function can be expressed as a sum of a few terms [69]. The HGPF used in the D-O model can be expanded in a cosine series, whose coefficients are expressed in terms of Legendre functions [11]. This allows a relatively quick solutions for the radiative transfer equations. However, as pointed out before (Sections 4.4 and 5.2), the use of the HGPF in tissue optics is questionable in terms of its effects on the accuracy and predictability of the simulations.

The D-O model lacks any form of experimental validation [78], and its predictability cannot be verified. Its suitability to render believable images of human skin was assessed by comparing its resulting images with images rendered using a simple Lambertian model. The differences were noticeable, but they were not as dramatic as one might expect from comparing images rendered using models with such different levels of complexity.

\section{The Diffusion Theory Based Model}

\subsection{Overview}

Jensen et al. [45] proposed a model for simulating the appearance of subsurface light transport in diffusive materials in which the scattering simulation algorithm were based on the diffusion theory (Section 4.2). In their model, henceforth referred to as D-T model, the general concept of the BSSRDF (bidirectional scattering-surface reflectance distribution 
function) [65] was used to describe the transport of light from one point on a surface to another. The performance of the D-T model was later improved by introducing a two-pass hierarchical algorithm [44].

Based on their observations, Jensen et al. [45] theorized that due to the effects of repeated multiple scattering, the light distribution tends to become symmetric (equal in all directions) and blurred in highly scattering media. Since the diffusion theory does not have a general analytical solution for finite media, they modeled subsurface reflection as a semiinfinite medium. They used a diffusion approximation for isotropic media called the dipole method developed by Eason [23] and Farrell et al. [25]. In the dipole method, two point sources are placed relative to the surface, one the positive real light located below the surface and the other a negative virtual light positioned above the surface. Using this method, they computed an analytical expression for the radiant exitance at some point from the incident flux at another point.

The D-T model has four input parameters: the absorption coefficient, the reduced scattering coefficient, the diffuse reflectance and the index of refraction. In order to determine the values of these parameters for various materials, they used a 3-CCD video camera to observe the radiant exitance across the surface of the material. They then used diffusion theory to compute the absorption coefficient and reduced scattering coefficient. In their follow-up work, Jensen et al. [44] reduce the space of parameters of the D-T model to the diffuse reflectance and an average scattering distance.

\subsection{Strengths and Limitations}

The usual assumption made in tissue optics that light entering a material leaves the material at the same position is relaxed in the D-T model. From a theoretical point of view, this is a valid contribution since such an assumption fails to represent the real behavior of diffusive or translucent materials. In practice, however, the effects of this assumption on the appearance of the materials may not be as significant as the effects resulting from other assumptions such as the homogeneity of the materials. Furthermore, the errors introduced in a scattering simulation by the the elimination of the positional argument of the BSSRDF may be diluted or compensated when one applies stochastic simulation approaches.

The D-T model is relatively simple to implement, general (can be used for different diffusive materials), and it is not as computationally expensive as Monte Carlo based models. In addition, it can be used to render visually pleasing images. These reasons may have motivated its incorporation (or some variant) in many commercial rendering packages. However, similarly to the H-K model, it presents some limitations associated to its generality. It does not take into account properties specific to organic materials. Also, like the H-K model, the input parameters come either from inversion procedures or can be arbitrarily set by the user 
(in the case of the simplified set of parameters [44]). Due to the fact that spectral properties such as the diffuse reflectance are actually input parameters to the model, it shall be classified only as a scattering model.

The D-T model considers the entire skin structure as one medium. As described in Section 3, skin is heterogeneous and layered, with each of the layers having different biological and optical properties (particularly the epidermis and dermis). It is reasonable to assume that diffusion approximation can be applied to simulating subsurface reflection in the dermis [99], however, there are some issues regarding its use for other skin layers. First, the diffusion approximation is not suitable when the scattering is mostly in the forward direction [28, 32, 109]. As mentioned in Section 3, the measurements performed by Bruls and van der Leun [9] demonstrate that both the stratum corneum and the epidermis tissues are highly forward scattering media. Second, as mentioned in Section 4.2, the diffusion theory is not applicable when the absorption coefficient is not significantly smaller than the scattering coefficient for turbid media [74, 81, 109]. Recall that human skin is characterized by the presence of pigments, such as melanin particles, which have a significant absorption cross section [12].

The evaluation of the D-T model and its variant is also based solely on visual inspection. There is no comparison to actual BRDF and BTDF values of any organic (or inorganic) material. An image of a human face was generated using the model to illustrate its suitability to render believable images. We remark that the model input parameters were obtained using an inversion procedure based on the use of video camera and diffusion theory. The image was compared to an image rendered using a simple Lambertian BRDF model. As expected, differences are noticeable.

\section{The Biophysically-Based Spectral Model}

\subsection{Overview}

Many models used in computer graphics rely on spectral parameters, such as reflectance and transmittance, whose values are either arbitrarily set by the user or obtained from direct measurements or inversion procedures. There are measured reflectance curves for human skin available in the biomedical literature, but they are limited to a very specific set of skin types and restricted to a narrow range of illuminating and viewing angles. Furthermore, measured transmittance curves for the skin organ as a whole are scarce. These aspects highlight the need to develop models of light interaction with human skin which can compute not only its scattering properties (given in terms of BRDF and BTDF), but also its spectral properties (given in terms of reflectance and transmittance). The biophysically-based spectral model (BioSpec) was proposed by Krishnaswamy and Baranoski [51] to address this need. 
The BioSpec model uses Monte Carlo based algorithms to simulate the processes of light propagation (surface reflection, subsurface reflection and transmission) and absorption in the skin tissues. It considers the stratification of skin into four semi-infinite main layers: stratum corneum, epidermis, papillary dermis, and reticular dermis. The model parameter space includes: the refractive index and thickness of each layer, the refractive index and the diameter of collagen fibrils, the extinction coefficient (Figure 3), concentration, and volume fraction of the main chromophores present in the skin tissues (eumelanin, phaeomelanin, oxyhemoglobin, deoxyhemoglobin, $\beta$-carotene and bilirubin) and the aspect ratio of the stratum corneum folds.

The propagation of light in the skin tissues is simulated by the BioSpec model as a random walk process using ray optics [6]. In this random walk process, the transition probabilities are associated with Fresnel coefficients computed at each interface between the layers, and the termination probabilities are determined by the ray free path length. Once a ray hits a skin specimen at the air $\Leftrightarrow$ stratum corneum interface, it can be reflected back or refracted into the stratum corneum. In the former case, the Biospec model computes the distribution of the reflected light taking into account the aspect ratio (or oblateness) of the stratum corneum folds (Section 3.2), and perturbing the reflected rays using a warping function based on a surface-structure function proposed by Trowbridge and Reitz [88], which represents rough air-material interfaces using microareas randomly curved. In the latter case, the ray can be reflected and refracted multiple times within the skin layers before it is either absorbed or propagated back to the environment thru the air $\Leftrightarrow$ stratum corneum interface. Recall that the subcutaneous tissue is a highly reflective medium (Section 3.2). Hence, for body areas characterized by the presence of hypodermis, the BioSpec models assumes total reflection at the reticular dermis $\Leftrightarrow$ hypodermis interface.

In the epidermis and stratum corneum, scattering is simulated using angular displacements measured by Bruls and van der Leun [9]. Since scattering quickly becomes diffuse in the dermal layers (Section 3.2), the Biospec model uses a cosine distribution to perturb the rays traveling in these layers. Rayleigh scattering is also computed in the dermal layers. Once a ray has been scattered, it is tested for absorption. The absorption testing is performed probabilistically every time a ray starts a run in a given layer, and consists in estimating the ray free path length using an expression based on Beer's law [91] and considering the extinction coefficient, concentration and volume fraction of the natural pigments found in the skin tissues.

\subsection{Strengths and Limitations}

BioSpec, to the best of our knowledge, is the first computer graphics model capable of computing both scattering and spectral quantities for skin specimens. Its implementation based on standard Monte Carlo methods enables its straightforward integration in a variety 
of rendering frameworks. The algorithmic simulations performed by the model, however, are time consuming and may represent a bottleneck in an image synthesis pipeline. Alternatively, these simulations can be run off-line, and the quantities computed by the model stored and reconstructed during rendering.

Although the simulation of surface reflection performed by the BioSpec model accounts for biological factors and employs a closer approximation to the skin mesostructures' aspect ratio than approaches based on the use of microfacets $[36,78]$, its generalization requires a more rigorous mathematical treatment. Similarly to previous models [36, 64, 45], shadowing and masking effects are not taking into account by the BioSpec model. Furthermore, we remark that BioSpec is a data driven model. As more data becomes available for the scattering properties of various skin layers or for the spectral properties of additional chromophores, the accuracy of the BioSpec model will increase. However, a lack of data can also result in limiting the skin conditions that can be simulated by this model.

Despite the limitations mentioned above, the scattering and spectral curves generated by the BioSpec model present a good quantitative and qualitative agreement with measured data. To date, Biospec is the only computer graphics model of light interaction with human skin to be evaluated through comparisons with spectral and scattering measured data, making it one of the few predictive models available in the computer graphics literature. Besides the experimental evaluation, Krishnaswamy and Baranoski [51] also rendered images to illustrate the applicability of the BioSpec model in the spectral simulation of medical conditions, e.g., jaundice (yellowish hue) and erythema (redness), associated with changes in the biophysical parameters, and to highlight an aspect for which measured data is scarce, namely the translucency of skin tissues (Figure 4). The transmission of light through the whole skin can be observed (in vivo) in body parts with a thin or absent hypodermis, such as ears, eye lids and fingers. In these areas the behavior of the transmitted light is near Lambertian, to the point where no internal structure can be noticeable [73].

\section{What's Next?}

In this tutorial, we have taken a broad view of the problems related to the simulations of light interaction with human skin. Many questions, however, remain to be answered. In this section, we examine a number of issues related to the improvement and evaluation of such simulations. We also look at more general open problems, their implications and recent developments in applied tissue optics. 


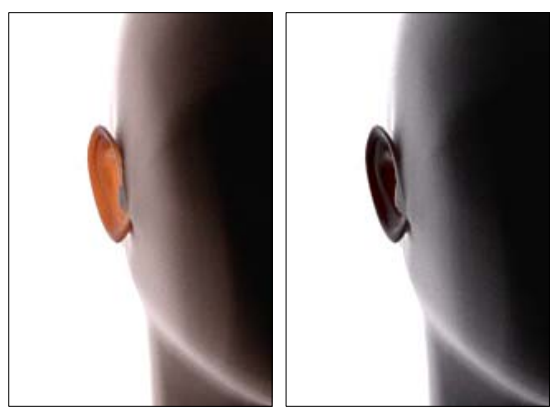

Figure 4. Images generated using the BioSpec model to show variations in the translucency of skin tissues associated with different levels of melanin pigmentation. Left: moderately pigmented specimen. Right: heavily pigmented specimen.

\subsection{Biological Input Data Availability}

Most models of light interaction with organic tissues available in the graphics literature are biologically motivated. Very few models, however, can be classified as biologicallybased, i.e., models that incorporate biological data and use algorithms based on the actual biological processes affecting the appearance of these tissues. Although these aspects are essential to establish the predictability of models, their complete fulfillment may present a logistics challenge, since the the most appropriate data may not available. For example, to compute spectral reflectance and transmittance for skin specimens, one uses the absorption spectra of various skin pigments which correspond to in vitro values, i.e., they are not measured in the tissues, but dissolved in a solvent. Since the exact position of the absorption peaks of the pigments depends on the solvents in which they are dissolved, and one can expect small shifts when comparing to in vivo values. Another example refers to refactive indices of organic materials. Ideally, one should use wavelength dependent quantities. In practice, most refractive indices available in the literature correspond to average values. Hence, a key issue that must be addressed to improve the predictability of skin optics simulations is the reliable measurement of biochemical properties, which will likely involve a substantial multidisciplinary effort.

\subsection{Accuracy vs. Undue Complexity}

The current models of light interaction with human skin do not account for the anisotropy ${ }^{9}$ of human skin, and usually the simulation of shadowing and masking effects

\footnotetext{
${ }^{9}$ The anisotropic behavior of a given material is observed when the material is rotated around its normal while the light and the viewer directions remain unchanged, and the light intensity reflected to the viewer varies.
} 
is not biologically-based. Although physically-based algorithms for the modeling of these phenomena with respect to inorganic materials have been proposed by computer graphics researchers [30], the generalization of these algorithms to organic materials is not straightforward since the simulation of the underlying biophysical processes involves the whole hierarchy of local illumination, i.e., issues at the microscopic (BRDF) mesoscopic (normal distribution) and macroscopic (geometry) levels need to be addressed. We believe that a careful modeling of skin's geometrical details [85] is essential to achieve a biophysically-based and predictable solution for these questions.

Accuracy and computational time are often conflicting issues in biophysically-based rendering. Although the main goal in this area is the design of accurate and efficient models, sometimes it is difficult to obtain this perfect combination. In order to achieve a higher level of accuracy, it may be necessary to add complexity to a model, which in turn may negatively affect its computational performance. However, this is not always the case. For example, light quickly becomes diffuse after the first interactions in the dermal layers (Section 3.2). In this case, a simple cosine distribution would be closer to the actual scattering profile of light transmitted through these layers than the use of the HGPF. Phase functions, such as the HGPF and its variations, were originally used in tissue subsurface scattering simulations to fit data measured at specific wavelengths [9]. Since then, their application has been extended to different organic materials despite the lack of supporting measured data and the fact that their parameters have no biological meaning. A recent investigation presented by Baranoski et al. [5] demonstrates that the use of a data oriented approach instead of the HGPF increases the accuracy/time ratio of algorithmic subsurface scattering simulations, and contributes to their predictability since the simulations are not controlled by arcane parameters.

\subsection{Evaluation and Comparison Data Availability}

Usually models of light interaction with matter are evaluated by visually inspecting the images generated using such models. Clearly, such an evaluation may be biased by factors not directly related to the model. For example, the use of an accurate geometric model of the body part being rendered and a careful post-processing spectral tone reproduction [8] may improve the realistic appearance of rendered skin specimens. These aspects, however, correspond to different stages of the rendering pipeline which should be evaluated independently

A current trend is to perform comparisons between model readings and measured data so that the models can be used in a predictive manner [35]. For example the BioSpec model was tested as a separated unit of the rendering pipeline and the results were compared with actual measured data $[24,58,101]$. These comparisons were performed using a virtual spectrophotometer and a virtual goniophotometer (Section 2), and reproducing the actual measurement conditions as faithfully as possible. 
This evaluation approach, however, is bounded by the availability of measured data. As mentioned earlier, skin reflectance and transmittance curves can be found in the biomedical literature, but they are usually restricted to a narrow range of measurement conditions. Furthermore, high fidelity comparisons of measured and modeled quantities require both qualitative and quantitative characterization data for the specimen at hand. Usually only qualitative characterization data is available for skin specimens used in tissue optics experiments. In terms of goniometric (BRDF and BTDF) curves, despite efforts of computer graphics researchers $[18,56,58,57]$, there is still a shortage of data, and the available data sets present the same limitations outlined for the reflectance and transmittance data sets. The lack of measured data is even more serious with respect to subsurface scattering since, to the best of our knowledge, the only relatively reliable data set corresponds to the measurements performed by Bruls and van der Leun [9], which are limited to two wavelengths in the visible range. Therefore, substantial efforts should be also directed towards the reliable measurement of multispectral surface and subsurface skin data.

\subsection{Biomedical Applications}

As mentioned earlier, computer simulations can assist the visual diagnosis of different skin conditions and diseases [91], including skin tumors [17, 16], and the indirect (noninvasive) measurement of the optical properties of skin tissues. These applications involve the non-iterative and the iterative use of a theoretical model of light interaction with these tissues [13]. The non-iterative approach consists in inverting the model (Section 4). In an iterative approach, the optical properties are implicitly related to measured quantities (e.g., reflectance and transmittance). The model is then run iteratively until these quantities are matched. Clearly, the predictability of these simulations is tied to the accuracy of the model being used. This aspect emphasizes the importance of evaluating the models both qualitatively and quantitatively. This tutorial has shown that no single model is superior in all aspects involved in the simulation of light interaction with human skin, and very few models have been properly evaluated. Hence, there is still room for improvement in this area.

Besides tackling the current limitations of the current computer graphics models and improving their evaluation, it will also be necessary to incorporate time dependent mechanisms of photon reemission in their formulation, and to extend their space of parameters to the non-visible regions of the light spectrum. Important biophysical processes are triggered by radiation hitting the skin at these wavelengths. For example, ultraviolet light can induce processes such as erythema (an abnormal redness of the skin caused by a dilation of the blood vessels followed by an increase in the volume fraction of blood in the dermal layers), melanogenesis (melanin production) and photoaging (discoloration and wrinkle formation) [49, 50, 96, 68]. The ultraviolet electromagnetic radiation (light) can be divided into three regions: UV-A (ranging from $320 \mathrm{~nm}$ to $380 \mathrm{~nm}$ ), UV-B (ranging from $280 \mathrm{~nm}$ to $320 \mathrm{~nm}$ ) and 
UV-C (ranging from 100nm to $280 \mathrm{~nm}$ ). The UV-C is mostly absorbed by the ozone layers in the atmosphere. UV-B penetrates deeper than UV-C in skin layers, and it may increase the melanogenesis after a certain period (6-8 hours) that follows the erythema reaction [50]. UV-A penetrates deeper than UV-B, and it can induce epidermal pigmentation immediately following exposure [50]. There are only few substances that can absorb UV-A efficiently [80]. Although the lack of UV-B in infants and small children may lead to disruption of bone growth and increase the probability of tooth decay [80], overexposure to ultraviolet radiation can induce the formation of melanomas [26] and carcinomas [68], the first and the second most common forms of skin cancer respectively. Melanoma is also the most serious form of skin cancer since it presents high metastatic potential and low cure rates [26].

\section{Conclusion}

In this tutorial, we presented an overview of the state of the art in the simulation of light interaction with human skin from a computer graphics perspective. Although that are several aspects beyond the scope of this work, such as geometrical modeling and tone reproduction, that also affect the realistic appearance of virtual humans, we believe that the predictability of rendering frameworks can be increased by bring more biological knowledge and data to bear on the modeling of light interaction with organic materials. Such an approach will likely extend the range of scientific applications of computer graphics algorithms as appropriately suggested by by Donald Greenberg in his Steve Coons Award speech [34]:

If computer graphics is to have a role in improving the future of our civilization, the real value will be in its application to science, engineering and design.

\section{Acknowledgements}

Thanks are due to the anonymous reviewers for their useful insights. The authors are also grateful for funding through the National Sciences and Engineering Council of Canada (NSERC Grant 213281) and the Canada Foundation for Innovation (CFI Project 6218).

\section{References}

[1] S. Alaluf, U. Heinrich, W. Stahl, H. Tronnier, and S. Wiseman. Dietary carotenoids contribute to normal human skin color and uv photosensitivity. Journal of Nutrition, 132:399-403, 2002.

[2] R.R. Anderson and J.A. Parrish. The optics of human skin. Journal of Investigative Dermatology, 77(1):13-19, 1981. 
[3] Elli Angelopoulou. Understanding the color of human skin. In Human Vision and Electronic Imaging VI, pages 243-251. SPIE, vol. 4299, 2001.

[4] S. Aydinli and H. Kaase. Measurement of luminous characteristics of daylighting materials. Technical Report IEA SHCP TASK 21 / ECBCS ANNEX 29, Institute of Electronics and Lighting Technology, Technical University of Berlin, September 1999.

[5] G.V.G. Baranoski, A. Krishnaswamy, and B. Kimmel. Revisiting the foundations of subsurface scattering. Technical Report CS-2003-45, School of Computer Science, University of Waterloo, December 2003.

[6] G.V.G. Baranoski and J.G. Rokne. An algorithmic reflectance and transmittance model for plant tissue. Computer Graphics Forum (EUROGRAPHICS Proceedings), 16(3):141-150, September 1997.

[7] G.V.G. Baranoski, J.G. Rokne, and G. Xu. Virtual spectrophotometric measurements for biologically and physically-based rendering. The Visual Computer, 17(8):506-518, 2001.

[8] I.E. Bell and G.V.G. Baranoski. More than RGB: moving toward spectral color reproduction. ACM SIGGRAPH Course Notes, San Diego, CA, July 2002. Course 24.

[9] W.A.G. Bruls and J.C. van der Leun. Forward scattering properties of human epidermal layers. Photochem. Photobiol., 40:231-242, 1984.

[10] R.L. Burden and J.D. Faires. Numerical Analysis. PWS-KENT Publishing Company, Boston, fifth edition, 1993.

[11] S. Chandrasekhar. Radiative Transfer. Dover Publications Inc., New York, 1960.

[12] M.R. Chedekel. Photophysics and photochemistry of melanin. In M.R. Chedekel L. Zeise and T.B. Fitzpatrick, editors, Melanin: Its Role in Human Photoprotection, pages 11-22, Overland Park, Kansas, USA, 1995. Valdenmar Publishing Company. 2223b.

[13] W. Cheong, S.A. Prahl, and A.J. Welch. A review of the optical properties of biological tissues. IEEE Journal of Quantum Electronics, 26(12):2166-2185, December 1990.

[14] D.Y. Churmakov, I.V. Meglinsky, S.A. Piletsky, and D.A. Greenhalgh. Analysis of skin tissues spatial fluorescence distribution by the Monte Carlo simulation. Journal of Physics D: Applied Physics, 36:1722-1728, July 2003.

[15] R.L. Cook and K.E. Torrance. A reflectance model for computer graphics. ACM Transactions on Graphics, 1(1):7-24, January 1982.

[16] S.D. Cotton. A noninvasive skin imaging system. Technical Report CSR-97-03, School of Computer Science, The University of Birmingham, 1997.

[17] S.D. Cotton and E. Claridge. Developing a predictive model of skin colouring. In SPIE Vol. 2708, Medical Imaging 1996, pages 814-825, 1996.

[18] K.J. Dana, B. van Ginneken, S.K. Nayar, and J.J. Koenderink. Reflectance and texture of real world surfaces. ACM Transactions on Graphics, 18(1):1-34, 1999.

[19] B.L. Diffey. A mathematical model for ultraviolet optics in skin. Physics in Medicine and Biology, 28(6):647-657, 1983. 
[20] M. Doi and S. Tominaga. Spectral estimation of human skin color using the Kubelka-Munk theory. In SPIE/IS\&T Electronic Imaging, pages 221-228. SPIE, vol. 5008, 2003.

[21] R.M.P. Doornbos, R. Lang, M.C. Aalders, F.W. Cross, and H.J.C.M. Sterenborg. The determination of in vivo human tissue optical properties and absolute chromophore concentrations using spatially resolved steady-state diffuse reflectance spectroscopy. Physics in Medicine and Biology, 44:967-981, 1999.

[22] ASTM Standard E284-91C. Standard terminology of appearance. In L.B. Wolff, S.A. Shafer, and G.E. Healey, editors, Physics-Based Vision Principles and Practice: Radiometry, pages 146-161, Boston, 1992. Jones and Bartlett Publishers.

[23] G. Eason, A. Veitch, R. Nisbet, and F. Turnbull. The theory of backscattering of light by blood. Journal of Physics, 11:1463-1479, 1978.

[24] M.A. Everett, E. Yeargers, R.M. Sayre, and R.L. Olsen. Penetration of epidermis by ultraviolet rays. Photochemistry and Photobiology, 5:533-542, 1966.

[25] T.J. Farell, M.S. Patterson, and B. Wilson. A diffusion theory model of spatially resolved, steadystate diffuse reflectance for the noninvasive determination of tissue optical properties in vivo. Medical Physics, 19:879-888, 1992.

[26] T.B. Fitzpatrick and J.L. Bolognia. Human melanin pigmentation: role in pathogenesis of cutaneous melanoma. In M.R. Chedekel L. Zeise and T.B. Fitzpatrick, editors, Melanin: Its Role in Human Photoprotection, pages 177-182, Overland Park, Kansas, USA, 1995. Valdenmar Publishing Company.

[27] R. Flewelling. Noninvasive optical monitoring. In J.D. Bronzino, editor, The Biomedical Engineering Handbook, pages 1-11, Boca Raton, FL, USA, 1981. IEEE Press. Section 86.

[28] Stephen T. Flock, Michael S. Patterson, Brian C. Wilson, and Douglas R. Wyman. Monte Carlo modeling of light propagation in highly scattering tissues - I: Model predictions and comparison with diffusion theory. IEEE Transactions on Biomedical Engineering, 36(12):1162-1168, December 1989.

[29] S.C. Foo. A gonioreflectometer for measuring the bidirectional reflectance of material for use in illumination computation. Master's thesis, Cornell University, August 1997.

[30] A. Fournier. From local to global illumination and back. In P. M. Hanrahan and W. Purgathofer, editors, Rendering Techniques'95 (Proceedings of the Sixth Eurographics Rendering Workshop), pages 127-136, Dublin, June 1995. Springer-Verlag.

[31] T. Fourty, F. Baret, S. Jacquemoud, G. Schmuck, and J. Verdebout. Leaf optical properties with explicit description of its biochemical composition: Direct and inverse problems. Remote Sensing of Environment, 56:104-117, 1996.

[32] K. Furutso. Diffusion equation derived from space-time transport equation. Journal of the Optical Society of America, 70:360, 1980.

[33] A.S. Glassner. Principles of Digital Image Synthesis. Morgan Kaufmann Publishers, Inc, San Francisco, 1995.

[34] D.P. Greenberg. 1987 Steven A. Coons Award Lecture. Computer Graphics, 22(1):7-14, February 1988. 
[35] D.P. Greenberg, J. Arvo, E. Lafortune, K.E. Torrance, J.A. Ferwerda, B. Walter, B. Trumbore, P. Shirley, S. Pattanaik, and S. Foo. A framework for realistic image synthesis. In SIGGRAPH, Annual Conference Series, pages 477-494, 1997.

[36] P. Hanrahan and W. Krueger. Reflection from layered surfaces due to subsurface scattering. In SIGGRAPH, Annual Conference Series, pages 165-174, August 1993.

[37] A. Haro, B. Guenter, and I. Essa. Real-time, photo-realistic, physically based rendering of fine scale human skin structure. In P. M. Hanrahan and W. Purgathofer, editors, Rendering Techniques'2001 (Proceedings of the 12th Eurographics Rendering Workshop), pages 53-62, London, June 2001. Springer-Verlag.

[38] L.G. Henyey and J.L. Greenstein. Diffuse radiation in the galaxy. Astrophysics Journal, 93:70 83, 1941.

[39] R.S. Hunter and R.W. Harold. The Measurement of Appearance. John Wiley \& Sons, New York, second edition, 1987.

[40] A. Ishimaru. Wave Propagation and Scattering in Random Media, volume 1. IEEE Press, New York, 2nd edition, 1978.

[41] S. Jacquemoud and S.L. Ustin. Leaf optical properties: A state of the art. In 8th International Symposium of Physical Measurements \& Signatures in Remote Sensing, pages 223-332, Aussois, France, January 2001. CNES.

[42] S.L. Jacques. Origins of tissue optical properties in the uva visible and nir regions. OSA TOPS on Advances in Optical Imaging and Photon Migration, 2:364-369, 1996.

[43] S.L. Jacques, C.A. Alter, and S.A. Prahl. Angular dependence of HeNe laser light scattering by human dermis. Lasers in Life Sciences, 1(4):309-333, 1987.

[44] H.W. Jensen and J. Buhler. A rapid hierarchical rendering technique for translucent materials. In SIGGRAPH, Annual Conference Series, pages 576-581, July 2002.

[45] H.W. Jensen, S.R. Marschner, M. Levoy, and P. Hanrahan. A practical model for subsurface light transport. In SIGGRAPH, Annual Conference Series, pages 511-518, August 2001.

[46] Z. Jin and K. Stammes. Radiative transfer in nonuniformly refracting layered media: atmosphere-ocean system. Applied Optics, 33(3):431-442, January 1994.

[47] D.B. Judd and G. Wyszecki. Color in Business, Science and Industry. John Wiley \& Sons, New York, third edition, 1975.

[48] K.F. Karner, H. Mayer, and M. Gervautz. An image based measurement system for anisotropic reflection. Computer Graphics Forum (EUROGRAPHICS Proceedings), 15(3):119-128, August 1996.

[49] G. Kelfkens and J.C van der Leun. Skin temperature changes after irradiation with UVB or UVC: implications for the mechanism underlying ultraviolet erythema. Physics in Medicine and Biology, 34(5):599-608, 1989.

[50] N. Kollias. The spectroscopy of human melanin pigmentation. In M.R. Chedekel L. Zeise and T.B. Fitzpatrick, editors, Melanin: Its Role in Human Photoprotection, pages 31-38, Overland Park, Kansas, USA, 1995. Valdenmar Publishing Company. 
[51] A. Krishnaswamy and G.V.G. Baranoski. A biophysically-based spectral model of light interaction with human skin. Computer Graphics Forum (EUROGRAPHICS Proceedings), 2004. To appear.

[52] A. Krishnaswamy, G.V.G. Baranoski, and Jon G. Rokne. Improving the reliability/cost ratio of goniophotometric measurements. Journal of Graphics Tools, 9(3):31-51, 2004.

[53] P. Kubelka and F. Munk. Ein beitrag zur optik der farbanstriche. Zurich Tech. Physik, 12:543, 1931.

[54] R. Lee, M.M. Mathews-Roth, M.A. Pathak, and J.A. Parrish. The detection of carotenoid pigments in human skin. Journal of Investigative Dermatology, 64:175-177, 1975.

[55] S.R. Marschner, H.W. Jensen, M. Cammarano, S. Worley, and P. Hanrahan. Light scattering from human hair fibers. ACM Transactions on Graphics, 22(3):780-791, 2003.

[56] S.R. Marschner, S. H. Westin, E.P.F. Lafortune, K.E. Torrance, and D.P. Greenberg. Imagebased brdf measurement. Technical Report PCG-99-1, Program of Computer Graphics, Cornell University, USA, January 1999.

[57] S.R. Marschner, S. H. Westin, E.P.F. Lafortune, K.E. Torrance, and D.P. Greenberg. Image-based brdf measurement including human skin. In D. Lischinski and G. W. Larson, editors, Rendering Techniques'1999 (Proceedings of the 10th Eurographics Rendering Workshop), pages 119-130, Granada, June 1999. Springer-Verlag.

[58] S.R. Marschner, S. H. Westin, E.P.F. Lafortune, K.E. Torrance, and D.P. Greenberg. Reflectance measurements of human skin. Technical Report PCG-99-2, Program of Computer Graphics, Cornell University, USA, January 1999.

[59] I.V. Meglinsky and S.J. Matcher. Modelling the sampling volume for skin blood oxygenation. Medical \& Biological Engineering \& Computing, 39:44-49, 2001.

[60] I.V. Meglinsky and S.J. Matcher. Computer simulation of the skin reflectance spectra. Computer Methods and Programs in Biomedicine, 70:179-186, 2003.

[61] N. Metropolis and S. Ulam. The Monte Carlo method. Journal of the American Statistical Association, 44(247):335-341, September 1949.

[62] J.R. Mourant, J.P. Freyer, A.H. Hielscher, A.A. Eick, D. Shen, and T.M. Johnson. Mechanisms of light scattering from biological cells relevant to noninvasive optical-tissue diagnostics. Applied Optics, 37(16):3586-3593, June 1998.

[63] H. Nakai, Y. Manabe, and S. Inokuchi. Simulation analysis of spectral distributions of human skin. In 14th International Conference on Pattern Recognition, pages 1065-1067, 1998.

[64] C.S. Ng and L. Li. A multi-layered reflection model of natural human skin. In Computer Graphics International 2001, pages 249-256, Hong Kong, July 2001.

[65] F.E. Nicodemus, J.C. Richmond, J.J. Hsia, I.W. Ginsberg, and T. Limperis. Geometrical considerations and nomenclature for reflectance. In L.B. Wolff, S.A. Shafer, and G.E. Healey, editors, Physics-Based Vision Principles and Practice: Radiometry, pages 94-145, Boston, 1992. Jones and Bartlett Publishers. 
[66] M. Nischik and C. Forster. Analysis of skin erythema using true-color images. IEEE Transactions on Medical Imaging, 16(6):711-716, December 1997.

[67] D. Parsad, K. Wakamatsu, A.J. Kanwar, B. Kumar, and S. Ito. Eumelanin and phaeomelanin contents of depigmented and repigmented skin in vitiligo patients. British Journal of Dermatology, 149:624-626, 2003.

[68] M.A. Pathak. Functions of melanin and protection by melanin. In M.R. Chedekel L. Zeise and T.B. Fitzpatrick, editors, Melanin: Its Role in Human Photoprotection, pages 125-134, Overland Park, Kansas, USA, 1995. Valdenmar Publishing Company.

[69] S.A. Prahl. Light Transport in Tissue. PhD thesis, The University of Texas at Austin, TX, USA, December 1988.

[70] S.A. Prahl, M. Keijzer, S.L. Jacques, and A.J. Welch. A Monte Carlo model of light propagation in tissue. SPIE Institute Series, IS 5:102-111, 1989.

[71] S.A. Prahl, M.J.C. van Gemert, and A.J. Welch. Determining the optical properties of turbid media using the adding-doubling method. Applied Optics, 32(4):559-568, 1993.

[72] S. Premoze. Analytic light transport approximations for volumetric materials. In Proceedings of the 10th Pacific Conference on Computer Graphics and Applications - Pacific Graphics'2002, pages 48-57, Beijing, October 2002.

[73] J. Rodriguez, I.V. Yaroslavsky, A.N. Yaroslavsky, H. Battarbee, and V.V. Tuchin. Time-resolved imaging in diffusive media. In V.V. Tuchin, editor, Handbook of Optical Biomedical Diagnostics, pages 357-404, Bellingham, WA, USA, 2002. SPIE Press.

[74] D.K. Sardar and L.B. Levy. Optical properties of whole blood. Lasers in Medical Science, 13:106-111, 1998.

[75] M. Shimada, Y. Yamada, M. Itoh, and T. Yatagai. Melanin and blood concentration in human skin studied by multiple regression analysis: assessment by Monte Carlo simulation. Physics in Medicine and Biology, 46:2397-2406, 2001.

[76] A.N. Shiryaev. Probability. Springer-Verlag, New York, second edition, 1996.

[77] C.R. Simpson, M. Kohl, M. Essenpreis, and M. Cope. Near infrared optical properties of exvivo human skin and subcutaneous tissues measured using the Monte Carlo inversion technique. Physics in Medicine and Biology, 43:2465-2478, 1998.

[78] J. Stam. An illumination model for a skin layer bounded by rough surfaces. In P. M. Hanrahan and W. Purgathofer, editors, Rendering Techniques'2001 (Proceedings of the 12th Eurographics Rendering Workshop), pages 39-52, London, June 2001. Springer-Verlag.

[79] K. Stamnes and P. Conklin. A new multi-layer discrete ordinate approach to radiative transfer in vertically inhomogeneous atmospheres. Journal of Quantum Spectroscopy and Radiative Transfer, 31(3):273-282, 1984.

[80] K. Stanzl and L. Zastrow. Melanin: an effective photoprotectant against UV-A rays. In M.R. Chedekel L. Zeise and T.B. Fitzpatrick, editors, Melanin: Its Role in Human Photoprotection, pages 59-63, Overland Park, Kansas, USA, 1995. Valdenmar Publishing Company. 
[81] J.M. Steinke and A.P. Shepherd. Diffusion model of the optical absorbance of whole blood. Journal of the Optical Society of America, 5(6):813-822, 1988.

[82] Y. Su, W. Wang, K. Xu, and C. Jiang. The optical properties of skin. In Optics in Health Care and Biomedical Optics: Diagnostics and Treatment, pages 299-304. SPIE, vol. 4916, 2002.

[83] J.L. Swerdlow. Unmasking skin. National Geographic, pages 36-63, November 2002.

[84] P.S. Talreja, G.B. Kasting, N.K. Kleene, W.L. Pickens, and T. Wang. Visualization of the lipid barrier and measurement of lipid pathlength in human stratum corneum. AAPS PharmSCi, 3(2):1-9, 2001.

[85] N. Thalmann, P. Kalra, J.L. Lévêque, R. Bazin, D. Batisse, and B. Querleux. A computational skin model: fold and wrinkle formation. IEEE Transactions on Information Technology in Biomedicine, 6(4):317-323, 2002.

[86] A.J. Thody, E.M. Higgins, K. Wakamatsu, S. Ito, S.A. Burchill, and J.M. Marks. Pheomelanin as well as eumelanin is present in human dermis. Journal of Investigative Dermatology, 97:340344, 1991.

[87] K.E. Torrance and E.M. Sparrow. Theory for off-specular reflection from roughned surfaces. Journal of the Optical Society of America, 57(9):1105-1114, 1967.

[88] T.S. Trowbridge and K.P. Reitz. Average irregularity representation of a rough surface for ray reflection. Journal of the Optical Society of America, 65(5):531-536, May 1975.

[89] N. Tsumura, M. Kawabuchi, H. Haneishi, and Y. Miyabe. Mapping pigmentation in human skin by multi- visible-spectral imaging by inverse optical scattering technique. In IS\&T/SID Eighth Color Imaging Conference, pages 81-84, 2000.

[90] N. Tsumura, N. Ojima, K. Sato, M. Shiraishi, H. Shimizu, H. Nabeshima, S. Akazaki, K. Hori, and Y. Miyake. Image-based skin color and texture analysis/synthesis by extracting hemoglobin and melanin information in the skin. In SIGGRAPH, Annual Conference Series, 2003.

[91] V.V. Tuchin. Tissue Optics Light Scattering Methods and Instruments for Medical Diagnosis. The International Society for Optical Engineering, Bellingham, WA, USA, 2000.

[92] V.V. Tuchin, S.R. Utz, and I.V. Yaroslavsky. Tissue optics, light distribution, and spectroscopy. Optical Engineering, 33:3178-3188, 1994.

[93] J.V. Uspensky. Introduction to Mathematical Probability. McGraw-Hill, New York, 1937.

[94] H.C. van de Hulst. Multiple Light Scattering: Tables, Formulas, and Applications, volume 1. Academic Press, New York, 1980.

[95] H.C. van de Hulst. Light Scattering by Small Particles. Dover Publications Inc., New York, 2nd edition, 1981.

[96] J.C. van der Leun. Ultraviolet Erythema. PhD thesis, University of Utrecht, The Netherlands, 1966.

[97] M.J.C. van Gemert, S.L. Jacques, H.J.C.M. Sterenborg, and W.M. Star. Skin optics. IEEE Transactions on Biomedical Engineering, 36(12):1146-1154, 1989.

[98] M.J.C. van Gemert and W.M. Star. Relations between the Kubelka-Munk and the transport equation models for anisotropic scattering. Laser in the Life Sciences, 1(4):287-298, 1987. 
[99] M.J.C. van Gemert, A.J. Welch, and W.M. Star. Tissue optics for a slab geometry in diffusion approximation. Laser in the Life Sciences, 2:295-302, 1987.

[100] B. van Ginneken, M. Stavridi, and J.J. Koenderink. Diffuse and specular reflectance from rough surfaces. Applied Optics, 37(1):130-139, 1998.

[101] M.J. Vrhel, R. Gershon, and L.S. Iwan. Measurement and analysis of object reflectance spectra. Color Research and Application, 19(1):4-9, 1994.

[102] S. Wan, R. Anderson, and J.A. Parrish. Analytical modeling for the optical properties of the skin with in vitro and in vivo appplications. Photochemistry and Photobiology, 34:493-499, 1981.

[103] L. Wang and S.L. Jacques. Hybrid method of Monte Carlo simulation and diffusion theory for light reflectance by turbid media. Optical Society of America, 10(8):1746-1752, 1995.

[104] G.J. Ward. Measuring and modeling anisotropic reflection. Computer Graphics (SIGGRAPH Proceedings), pages 265-272, July 1992.

[105] B.C. Wilson and G. Adam. A Monte Carlo model for the absorption and flux distributions of light in tissue. Medical Physics, 10:824-830, 1983.

[106] A.N. Yaroslavsky, A.V. Priezzhev, J. Rodriguez, I.V. Yaroslavsky, and H. Battarbee. Optics of blood. In V.V. Tuchin, editor, Handbook of Optical Biomedical Diagnostics, pages 169-216, Bellingham, WA, USA, 2002. SPIE Press.

[107] A.N. Yaroslavsky, S.R. Utz, S.N. Tatarintsev, and V.V. Tuchin. Angular scattering properties of human epidermal layers. In Human Vision and Electronic Imaging VI, pages 38-41. SPIE, vol. 2100, 1994.

[108] G. Yoon. Absorption and Scattering of Laser Light in Biological Media - Mathematical Modeling and Methods for Determining Optical Properties. PhD thesis, University of Texas at Austin, USA, 1988.

[109] G. Yoon, S.A. Prahl, and A.J. Welch. Accuracies of the diffusion approximation and its similarity relations for laser irradiated biological media. Applied Optics, 28(12):2250-2255, 1989.

[110] G. Yoon, A.J. Welch, M. Motamedi, and M.C.J. van Gemert. Development and application of three-dimensional light distribution model for laser irradiated tissue. IEEE Journal of Quantum Electronics, QE-23:1721-1733, 1987.

[111] G. Zonios, J. Bykowsky, and N. Kollias. Skin melanin, hemoglobin, and light scattering properties can be quantitatively assessed in vivo using diffuse reflectance spectroscopy. Journal of Investigative Dermatology, 117(6):1452-1457, 2001. 